\title{
Percutaneous retrieval of centrally embolized fragments of central venous access devices or knotted Swan-Ganz catheters. Clinical report of 14 retrievals with detailed angiographic analysis and review of procedural aspects
}

\author{
Łukasz Kalińczuk ${ }^{1}$, Zbigniew Chmielak², Artur Dębski ${ }^{2}$, Cezary Kępka ${ }^{1}$, Piotr N. Rudziński ${ }^{1}$, Sebastian Bujak ${ }^{1}$, \\ Mirosław Skwarek ${ }^{1}$, Andrzej Kurowski ${ }^{3}$, Zofia Dzielińska ${ }^{1}$, Marcin Demkow ${ }^{1}$ \\ ${ }^{1}$ Department of Coronary and Structural Heart Diseases, The Cardinal Stefan Wyszynski Institute of Cardiology, Warsaw, Poland \\ 2Department of Interventional Cardiology and Angiology, The Cardinal Stefan Wyszynski Institute of Cardiology, Warsaw, Poland \\ ${ }^{3}$ Department of Anesthesiology, The Cardinal Stefan Wyszynski Institute of Cardiology, Warsaw, Poland
}

Adv Interv Cardiol 2016; 12, 2 (44): 140-155 DOI: 10.5114 /aic.2016.59365

\begin{abstract}
Introduction: Totally implantable venous access systems (TIVAS), Swan-Ganz (SG) and central venous catheters (CVC) allow easy and repetitive entry to the central cardiovascular system. Fragments of them may be released inadvertently into the cardiovascular system during their insertion or as a result of mechanical complications encountered during long-term utilization.

Aim: To present results of percutaneous retrieval of embolized fragments of central venous devices or knotted SG and review the procedural aspects with a series of detailed angiographies.

Material and methods: Between January 2003 and December 2012 there were $14(\sim 0.025 \%)$ successful retrievals in 13 patients (44 \pm 16 years, $15 \%$ females) of embolized fragments of TIVAS $(n=10)$ or CVC $(n=1)$ or of dislodged guide-wires $(n=2)$ or knotted SG $(n=1)$.

Results: Foreign bodies with the forward end located in the right ventricle (RV), as well as those found in the pulmonary artery $(\mathrm{PA})$, often required repositioning with a pigtail catheter as compared to those catheter fragments which were located in the right atrium (RA) and/or great vein and possessed an accessible free end allowing their direct ensnarement with the loop snare $(57.0 \%$ $(4 / 7)$ vs. $66.7 \%(2 / 3)$ vs. $0.0 \%(0 / 3) ; p=0.074$ respectively). Procedure duration was $2-3$ times longer among catheters retrieved from the PA than among those with the forward edge located in the RV or RA (30 (18-68) vs. 13.5 (11-37) vs. 8 min (8-13); $p=0.054$ respectively). The SG catheter knotted in the vena cava superior (VCS) was encircled with the loop snare introduced transfemorally, subsequently cut at its skin entrance and then pulled down inside the $14 \mathrm{Fr}$ vascular sheath.

Conclusions: By using the pigtail catheter and the loop snare, it is feasible to retrieve centrally embolized fragments or knotted central venous access devices.
\end{abstract}

Key words: percutaneous retrieval, embolized fragments, knotted, central venous access devices.

\section{Introduction}

The necessity for the use of vasopressors, chemotherapy, and parenteral nutrition, as well as the need for hemodynamic monitoring (Swan-Ganz catheter - SG) or for extracorporeal therapies, and finally the demand for cardiac pacing, all require central venous access (central venous catheter - CVC). Central venous access is mandatory for long-term parenteral therapy, with common use of totally implantable venous access systems (TIVAS) [1-3]. These are designed for simple and repetitive entry to the venous system. Totally implantable venous access systems consist of a reservoir compartment (port), which as a whole is surgically placed in a subcutaneous pocket (usually in the upper chest). Its self-sealing silicone septum is directed towards the skin surface, allowing needle puncture of its rubber. The port is connected through a connector lock with the catheter, which is tunneled beneath the skin toward the cannulated central vein. The Seldinger technique is commonly used for the placement of CVC and TIVAS, and refers to guide-wire (GW) insertion

\section{Corresponding author:}

Łukasz Kalińczuk MD, PhD, Department of Coronary and Structural Heart Diseases, The Cardinal Stefan Wyszynski Institute of Cardiology, 42 Alpejska St, 04-628 Warsaw, Poland, phone: +48 505794 691, fax: +48 2234345 16, e-mail: lukasz.kalinczuk@gmail.com Received: 1.12.2015, accepted: 21.12.2015. 
into the lumen of a central vein, as a conduit for subsequent intravascular device placement. Errant positioning and resultant kinking of the GW, along with its withdrawal through the inserting needle, can result in accidental shear-off of the distal GW part, with its consequent central embolization into the caval vein, heart chamber or pulmonary vasculature. Either the subclavian or internal jugular vein, or infra- versus supra-clavicular site, and finally the left- or right-sided location, are used for CVC and TIVAS insertion. It creates a large diversity of arrangements, with inherent advantages and drawbacks but only little variation in long-term mechanical complications. On the other hand, a defective alignment between the TIVAS's port and catheter ('Cath-Lock mechanism'), as well as TIVAS insertion near the costoclavicular ligament exposing the catheter's section on a repetitive compression between the first rib and the clavicle (the pinch-off syndrome - POS), are risk factors of the catheter's rupture with its distal part central embolization $[4,5]$. Interestingly, the SG pulmonary artery catheter, which is soft and balloon-tipped to be inertly propelled by the flow of blood into the pulmonary artery (PA) avoiding fluoroscopic guidance, for the same reasons has an increased risk of accidental knotting. Guide-wire loss during insertion of the CVC occurs approximately twice in several thousand procedures [4]. The relative prevalence of distal embolization of a CVC catheter fragment $(0.1 \%)$ or part of the TIVAS catheter (up to $4 \%$ ), as well as the frequency of SG knotting (1 per 500 consecutively inserted but constituting $2 / 3$ of all reported knotted intravascular devices), is low [1, 6-9]. However, with their widespread usage, the absolute number of cases of distal embolization of their parts, as well as the frequency of SG knotting, is of high clinical importance. Furthermore, there is high incidence of bacterial contamination of intravenous polyethylene catheters left in place over $48 \mathrm{~h}(40.7 \%)$ compared to those remaining sterile if in situ for $<48 \mathrm{~h}$, and serious adverse events (including death) related to the distal foreign body's embolization have been reported. All of these factors necessitate their removal, even years after the event [10, 11]. In 1971 Dotter et al., for the first time, reviewed collected case reports documenting 100 cases of centrally embolized broken-off fragments of CVC or GW, of which 29 were retrieved with transluminal catheterization instead of surgery [12]. Nowadays, open cardiotomy and arteriotomy of the PA for retrieval of embolized catheters have been replaced solely by percutaneous techniques, using either dedicated or homemade devices.

\section{Aim}

The aim of our study was to report our experience with 14 percutaneous retrievals of centrally embolized fragments of central venous access devices or knotted SG catheters, along with detailed angiographic analysis and a discussion of procedural aspects.

\section{Material and methods}

At the single institution of the tertiary referral interventional cardiologic centre providing specialized services for affiliated health centers, a retrospective analysis was performed on 55858 consecutive percutaneous procedures (36 619 diagnostic and 19239 therapeutic procedures), performed from January 2003 to December 2012. There were 14 transcatheter retrievals in 13 patients identified, referred due to the diagnosis of a centrally embolized catheter fragment of TIVAS $(n=10)$ or CVC $(n=1)$, or embolized CVC guide-wire $(n=2)$ or knotted SG catheter $(n=1)$. There were 3 catheter fragments of Polysite Mini 3000 Series retrieved (Teleflex Medical Europe Ltd., Athlone, Co. Westmeath, Ireland), 3 fragments of PowerPort BARD (Bard Access Systems, Inc., Salt Lake City, USA), 2 fragments of Celsite access port (B. Braun Medical; B.P. 331; 92107 Boulogne; France), 2 fragments of Ambix Intraport (Fresenius Kabi AG, Bad Homburg, Germany) and 1 fragment of Broviac CVC (C. R. Bard, Inc., Murray Hill, New Jersey). Also, one complete and one fragment of a "J"-tip GW were retrieved, both from Hydrocath Assure CVC (Argon Critical Care Systems Singapore Pte. Ltd., Singapore). Also, a knotted standard 4-lumen SG catheter was retrieved (Edwards Lifesciences LLC, Irvine, CA, USA).

$A$ chest $X$-ray (CXR) was made immediately after the inadvertent disappearance of the entire GW encountered during an inappropriate handling of the introducer set $(n=1)$, as well as just after the acknowledgement that the large fragment of the "J"-tip GW was lost due to accidental severing on the needle's bevel during its inappropriate withdrawal through the needle $(n=1)$. Knotting of the SG was suspected when a proper PA waveform could not be obtained despite the numerous catheter maneuvers employed, along with insertion of more than $40 \mathrm{~cm}$ of its length, with final CXR confirmation.

In the case of embolized fragments of TIVAS, a common presentation of this was the difficulty in irrigation and with blood withdrawal as well as the resistance to fluids infusion, with a positive response to the patient position changes. In the above scenario CXR was done to define the exact mechanism of the complication and to locate a catheter fragment. All the foreign bodies were radio-opaque, and their angiographic appearance was carefully analyzed to identify the forward (initially most distal to the vein entrance) and the trailing edge (initially proximal to the vein entrance). The primary causes of foreign body embolization were attempted to be recognized based on a careful analysis of the catheter edge contour (sharp vs. distorted, in order to differentiate the occurrence of POS), and an analysis of alterations in the alignment site (searching for the 'Cath-Lock mechanism').

Patients were informed of potential complications including knotting, valve leaflet damage and the possibility of surgical intervention. Under local anesthesia the com- 
mon femoral vein was punctured and an $8 \mathrm{Fr}$ or larger standard sheath was inserted. In the case of all retrievals, primarily the custom-made loop snare was delivered to the region of interest with a 6 Fr JR 3.5 Launcher guiding catheter (Medtronic, Inc., Minneapolis, MN USA) to ensnare the free-end of the foreign body, which was then pulled down inside the vascular sheath or close to its tip and then retrieved outside in toto. There were 2 types of loop snares used: (1) the Amplatz GooseNeck Snare Kit (ev3 Endovascular, Inc., Plymouth, MN, USA), which is a mono-plane system with a super-elastic snare constructed of nitinol cable and a gold plated tungsten loop remaining true $90^{\circ}$ to the lumen of the guiding catheter, and (2) the Multi-Snare (pfm medical ag, Köln, Germany), with the unique design of its loop offering additional coaxial plane allowing lateral retrieval. These were available in a range of sizes (loop diameter of 15-3 and 4-40 mm, respectively), and their selection was at each operator's individual discretion. When the free end of the embolized catheter could not be identified, its prior reposition was performed using the $6 \mathrm{Fr}$ pigtail catheter (Boston Scientific Corporation, Marlborough, MA, USA), which was placed alongside the embolized catheter and rotated repeatedly to enfold it. Otherwise, it was passed above and coaxially to the migrated catheter along the 0.0889 $\mathrm{cm}$ guidewire, which was then withdrawn so as to bend the pigtail curve that hooked the catheter's mid-shaft, allowing it gentle manipulations aimed at liberating its free end. No heparin or antibiotics were given and no contrast medium was used. All procedures were fluoroscopy guided. The radiation exposures (median measurable dose area product, DAP), as well as procedural and fluoro durations, were all recorded on a prospective basis and evaluated thereafter from the radiology reporting database. The length of all the retrieved foreign bodies was measured and archived. Institutional review board exemption was obtained for retrospective review of procedure records and the patients' medical charts.

\section{Statistical analysis}

Categorical data are presented as frequencies and compared with Fisher's exact test. Normally distributed variables were compared with Student's $t$-test and are presented as mean \pm 1 SD. The Mann-Whitney and Kruskal-Wallis ANOVA tests were used for comparisons of variables with other than normal distribution; these are presented as medians with minimal and maximal values (min-max). A $p$ value $<0.05$ was considered significant. Analysis was performed with the SPSS 16.0 (SPSS, Inc., Chicago, IL).

\section{Results}

Two male and 11 female patients were studied, in whom 14 foreign bodies were retrieved, each during the single-staged procedure $(\sim 0.025 \%$ of all diagnostic and therapeutic interventions done during the 10-year-long study period). The basic patients' clinical characteristics and details on the type, location, length of the embolized foreign body, and relevant procedural data are presented in Table I. Patient \#5 complained of a recurrent twinge of chest pain, whereas patient \#8 suffered from sustained supraventricular tachyarrhythmia. The others were clinically asymptomatic for the embolization. The most common indication for CVC/TIVAS placement was neoplasm ( $n=9 / 13,69 \%)$. The majority of these catheters have the forward edge found in the RV (7/13, 54\%), whereas in the other 3 it was located in the RA $(3 / 13$, $23 \%$ ) and the other 3 catheter fragments of TIVAS were situated as a whole in the PA (the knotted SG catheter was pulled up into the VCS). All the foreign bodies were successfully removed using either the loop snare alone ( $n=8,57 \%$ ) or after their prior repositioning with the pigtail catheter $(n=6,43 \%)$. The foreign bodies with the forward edge located in the RV, as well as those found in the PA, need more often to be initially repositioned compared to those located in the RA or great vein $(57.0 \%$ $(n=4 / 7)$ vs. $66.7 \%(n=2 / 3)$ vs. $0.0 \% ; p=0.074$ respectively). The median length of retrieved foreign bodies was $14.9 \mathrm{~cm}$ (6.8-30.0). On average, there were insignificant length differences among catheters retrieved from the PA vs. those with the forward edge in the RV vs. those with the forward edge in the RA (12.7 (6.8-18.4) vs. 15.6 (6.9$22.0)$ vs. $14.4 \mathrm{~cm}(13.3-30.0) ; p=0.509$ respectively). The catheter fragments of TIVAS were shorter than the embolized GW fragments and the retrieved single CVC fragment (13.0 (6.8-20.2) vs. $26.0(22.0-30.0)$ vs. $19.1 \mathrm{~cm}$, $p=0.053$ respectively). The overall median procedure duration was $13.0 \mathrm{~min}$ (7-68), and it was on average $\sim 2$ times longer among catheters retrieved from the PA than among those with the forward edge in the RV vs. those with its location in the RA (18.0 (7-30) vs. 13.0 (11-68) vs. $8.0 \mathrm{~min}(8-13) ; p=0.230$ respectively). Procedure duration was on average more than 2 times longer if reposition was needed (30.0 $(12-68 ; n=5)$ vs. 12.0 min among procedures with no reposition (7-18; $n=9), p=0.060$ respectively). The longest procedures ( $\geq 30 \mathrm{~min}$ ) were those done in patients with foreign bodies anchored as a whole within cavities of the right heart and/or hepatic vein (patient \#5 and Figure 6; patient \#9 and Figure 11, respectively). The other longest procedure was in patient \#11, in whom an embolized $18-\mathrm{cm}$ long catheter without an apparent free end was found in the PA (Figure 13). The median fluoroscopic time was $6.1 \mathrm{~min}$ (0.47-42.0), and it was longer for catheters retrieved from the PA vs. those with the forward edge in the RV as compared to those with its location in the RA (6.3 (6-42) vs. $6.4(2.2-26.4)$ vs. $2.3 \min (0.5-6.1), p=0.225$ respectively). The median DAP was $575 \mu \mathrm{Gy} \cdot \mathrm{m}^{2}$ (25-8457); its value was lowest for the foreign bodies with the forward edge located in the RA vs. its location in the PA vs. its presence in the RV 


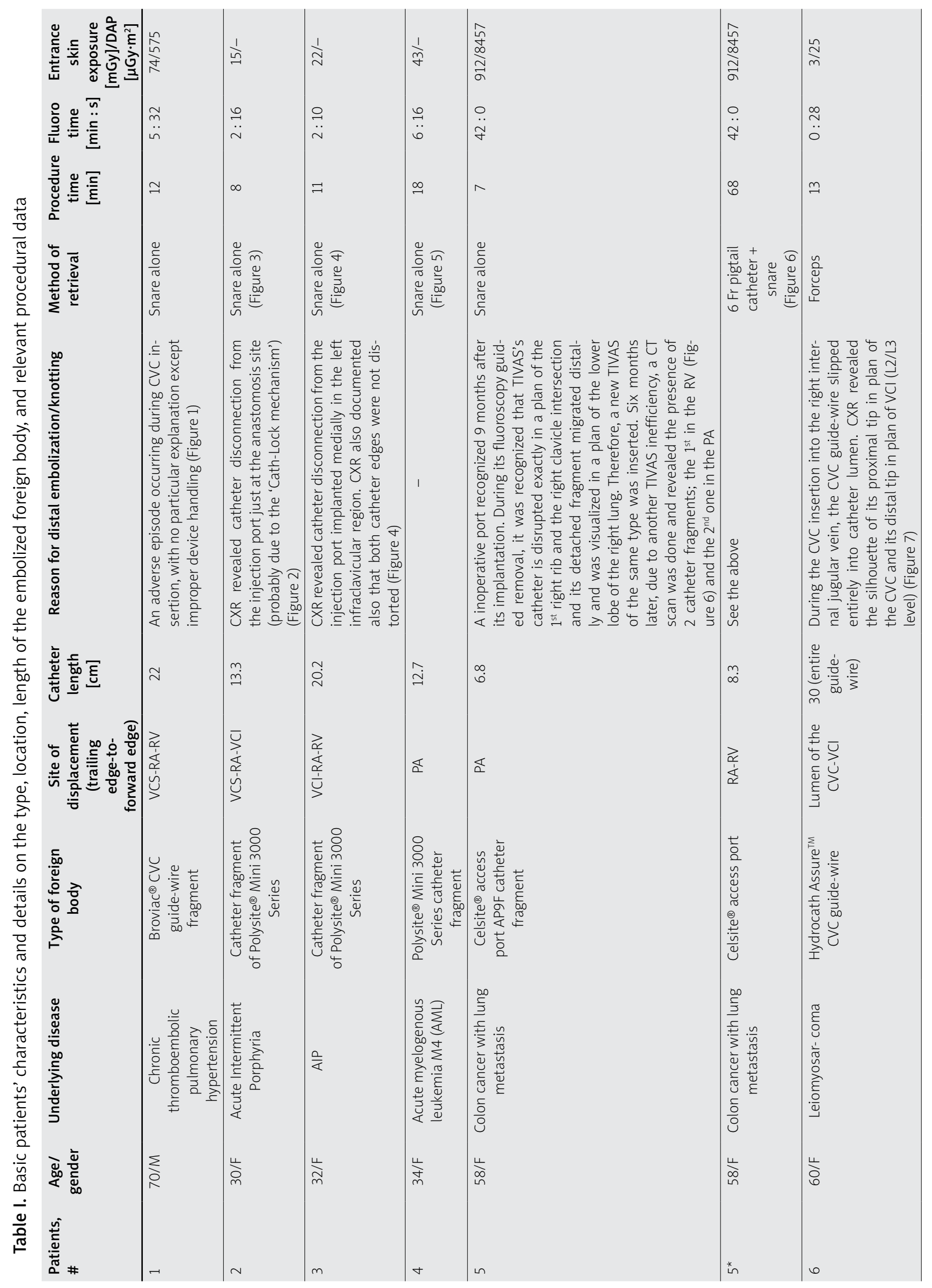




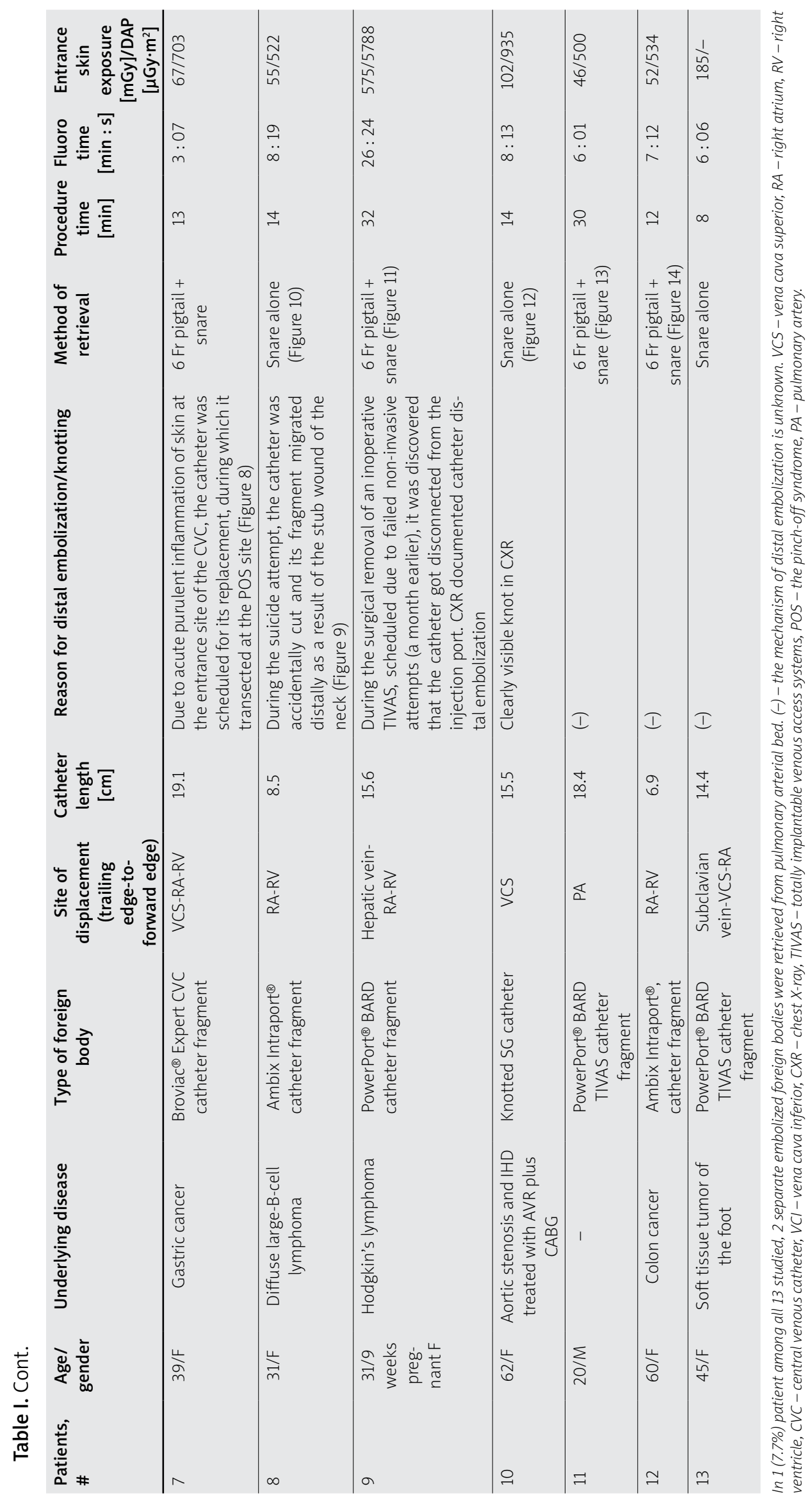



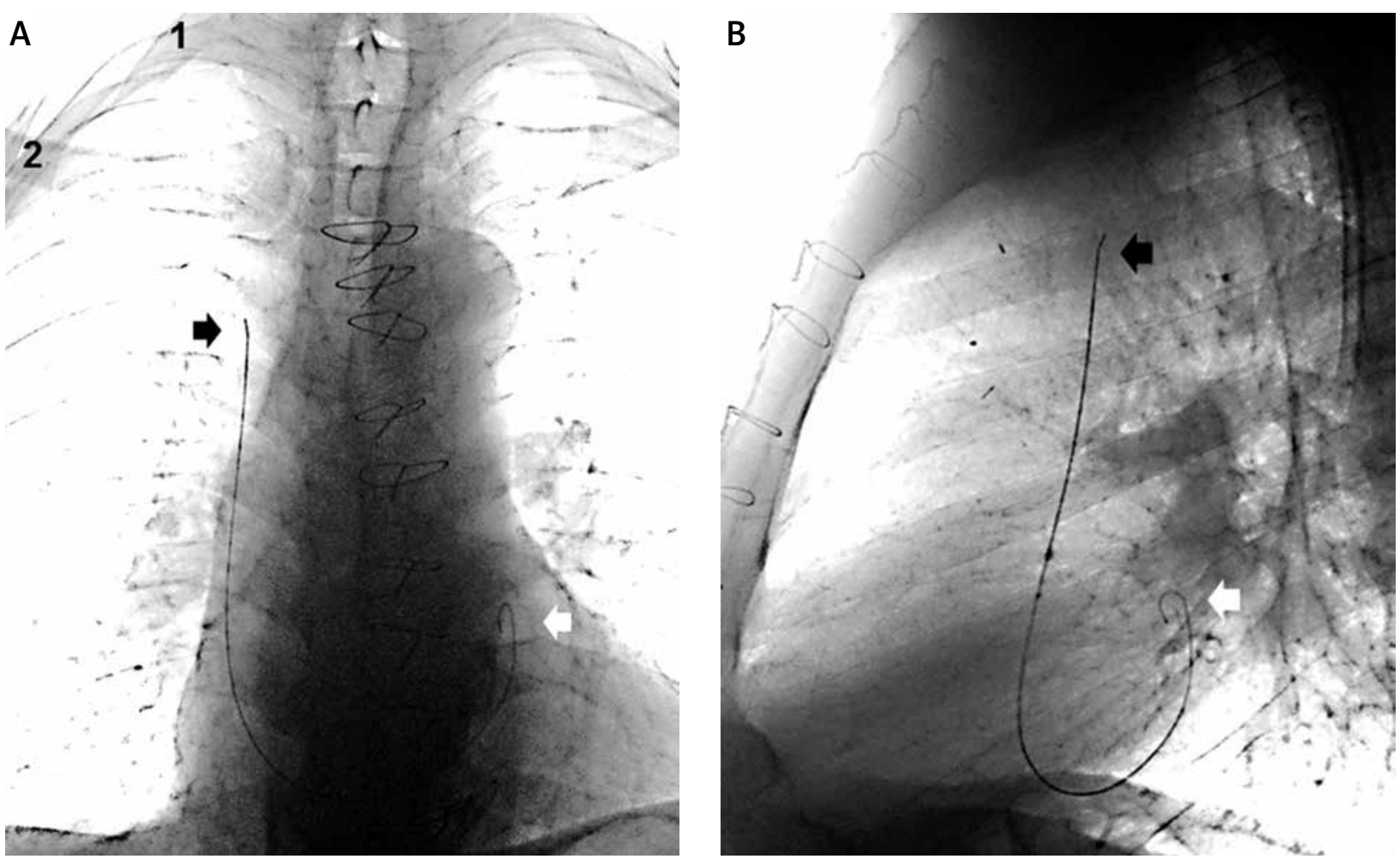

Figure 1. Digital CXR presented in inverse mode with subtracted view (A - anteroposterior and $\mathbf{B}$ - lateral view) taken due to resistance that occurred after a forceful and inadvisable GW withdrawal with an introducer needle still being inserted into the subclavian vein. It revealed embolization of a large GW fragment (Broviac) probably due to its shear off at the beveled needle tip. 1 - the $1^{\text {st }}$ right rib, 2 - the right clavicle, black arrow - indicates proximal end of the GW fragment, which has no contact with the skin surface and is located within the lumen of the VCS, white arrow - indicates "J" tip of the GW, engaged in the RV outflow tract

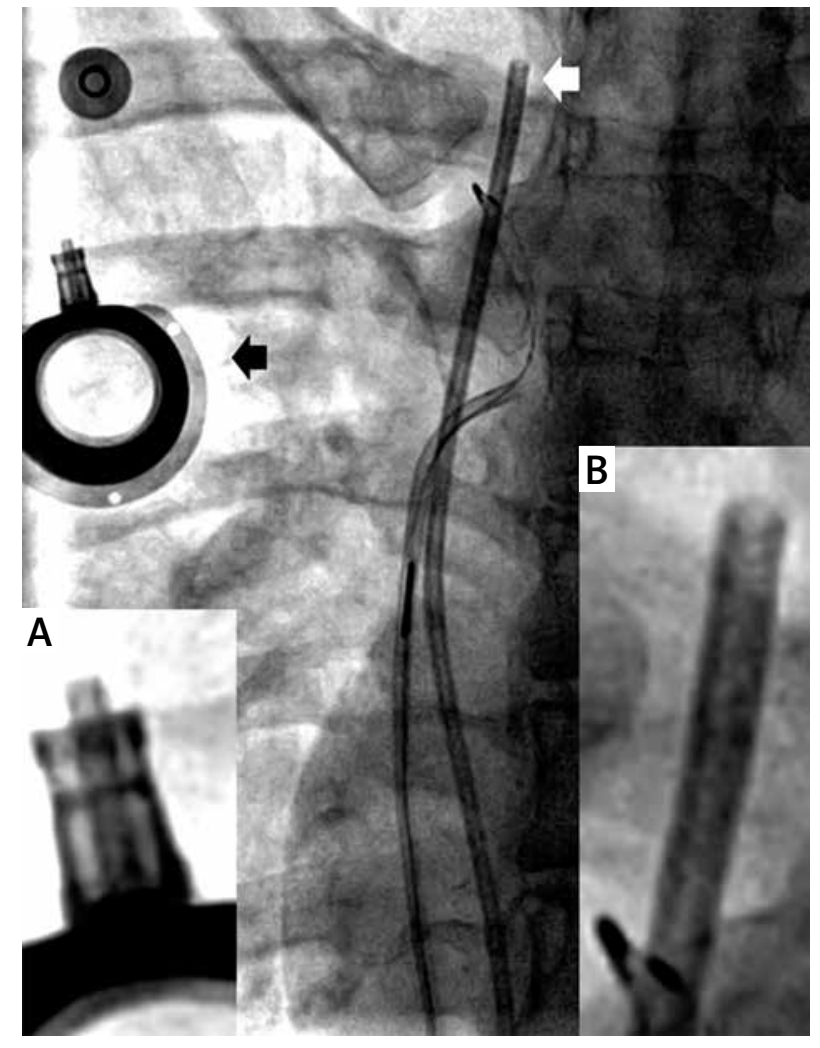

$\left(142,5\right.$ (25-1758) vs. 500 (409-8457) vs. $554.5 \mu \mathrm{Gy} \cdot \mathrm{m}^{2}$ (209-5788), $p=0.482$ respectively). Dose area product values were the same for the foreign bodies requiring prior reposition and those retrieved with the snare alone (534 (409-5788) vs. $548.5 \mu \mathrm{Gy} \cdot \mathrm{m}^{2}$ (25-8457), $p=0.833$ respectively). No thrombus formation was detected attached to the retrieved fragments, and none of these fragments were torn during the retrieval.

\section{Discussion}

Most aspects of the presented subject have been thoroughly investigated by previous scientists. The origi-

Figure 2. The Polysite Mini 3000 Series catheter intracardiac migration, with its trailing edge located in the right brachiocephalic vein (white arrow) and its forward edge lodged in the tricuspid annulus (Figure 3). CXR gave an insight into the most frequent mechanism of TIVAS's catheter distal embolization, relying on its disconnection from the injection port (black arrow) just at the anastomosis site ('Cath-Lock mechanism'). Note that both the port's ending and the catheter's proximal tip are not distorted (see the magnified pictures: $\mathbf{A}$ and $\mathbf{B}$, correspondingly) 

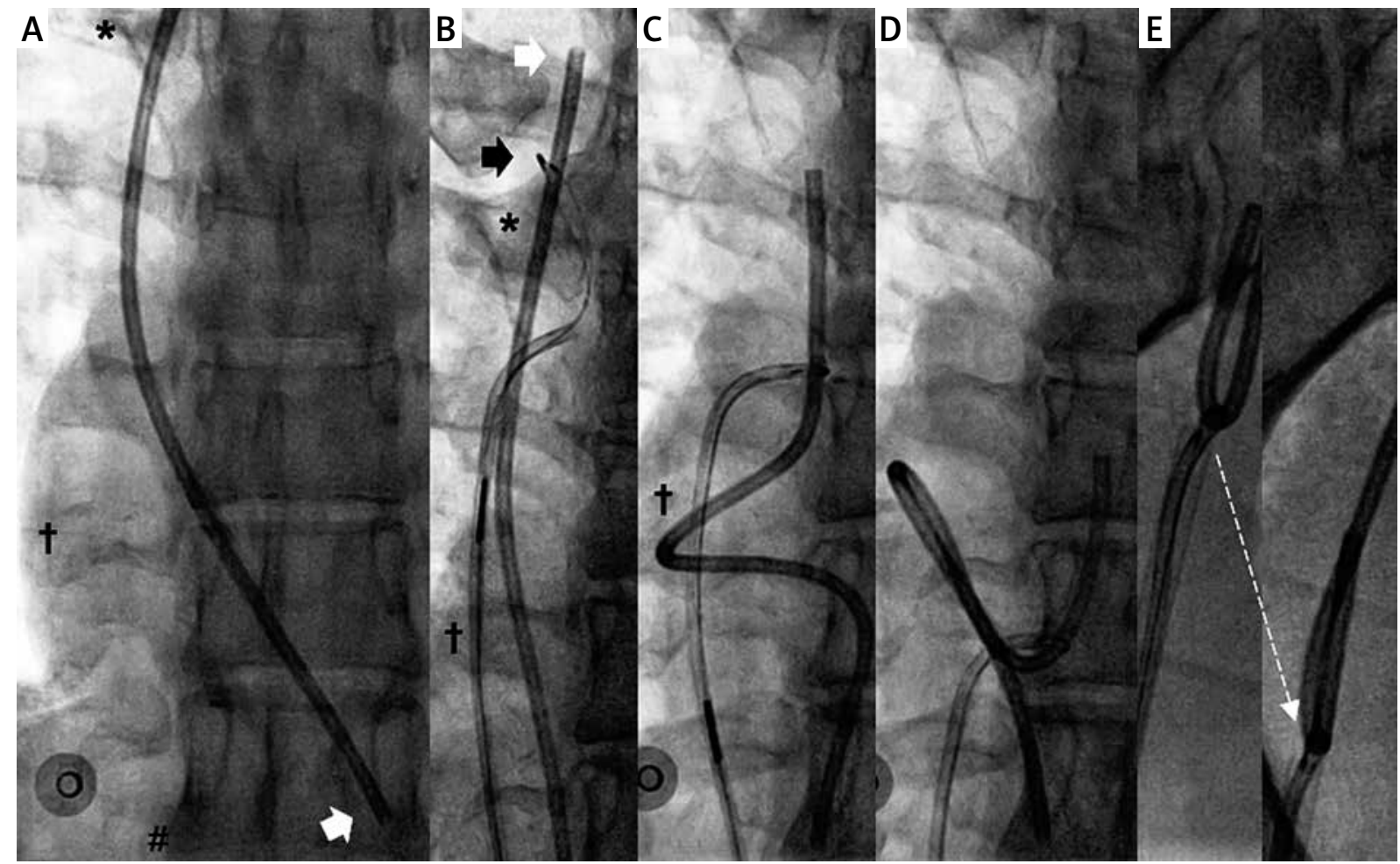

Figure 3. The forward catheter's edge (Polysite) was lodged in the tricuspid annulus (A, white arrow); however, in the VCS $\left(^{*}\right)$ the catheter's free end was identified (trailing edge, B, white arrow) allowing the Amplatz Nitinol goose-neck snare (black arrow) to be advanced transfemorally (from the right site) through the vena cava inferior ( $\mathrm{VCI})\left(^{\#}\right)$ and the RA $\left(^{+}\right)$, using a 6 Fr JR guiding catheter. When the foreign body was encircled by the loop, the snare was tightened and rotated by an angle of $180^{\circ}$ in the RA cavity (C-D) and then the catheter-snare complex was pulled down till it rested against the tip of a $10 \mathrm{Fr}$ vascular sheath, and then this arrangement, with the unsheathed catheter, was gently withdrawn in toto through the vessel wall upon the skin surface (E, dotted arrow)

nality of this article lies in it being the first presentation of Polish 'real life' practices in percutaneous retrieval of embolized fragments or knotted central venous access devices, supported with a series of detailed angiographies magnifying this practical insight.

Most of the presented percutaneous retrievals were on embolized TIVAS catheters. A retrospective analysis of 1500 consecutive patients, with a high proportion of TIVAS implantations for chemotherapy of solid tumors, showed an average catheter life of 284 days (range: 2-1563 days). Common complications reported after TIVAS in the above analysis were infections (4.8\%), thrombosis (3.2\%) and surprisingly low numbers of catheter fracture $(0.2 \%)$ or its disconnection $(0.2 \%)$, compared to those quoted in the introduction [1]. This might be explained by the fact that unlike those who routinely use the Seldinger technique, the quoted authors applied the cut-down technique for TIVAS insertion, relying on surgical isolation of the cephalic vein at the delto-pectoral groove and inserting the catheter through a small venotomy. In the authors' opinion, the low-cost open cut-down technique protects against the POS, as the catheter's passage between the first rib and the clavicle is ruled out [13]. At the same time, the authors who are advocates of the Seldinger technique indicate that for POS prevention, catheters should be inserted into the subclavian vein at the junction of the outer and middle thirds of the clavicle, just lateral to the thoracic outlet (lateral to the mid-clavicular line). The clinical manifestation of the POS syndrome is TIVAS functional occlusion, responding to the postural changes. Because raising the arms or shrugging opens the costoclavicular angle, the CXR to search for significant radiographic findings among patients with POS suspicion should be taken with the patient upright and then with the patient's arms by the side [7]. Furthermore, since TIVAS disruption occurs most frequently 0.5 to 1 year after its insertion, which is similar to the history of our patient \#5, it is recommended to perform CXR every half year to assess the grade (0-3) of angiographic severity of catheter distortion (Figure 8) [14, 15]. The other commonly reported location of the TIVAS catheter fracture is at the site of its anastomosis with the injection port (Figure 2) [2]. This particular place is exposed to repetitive bending forces, especially with medial TIVAS 


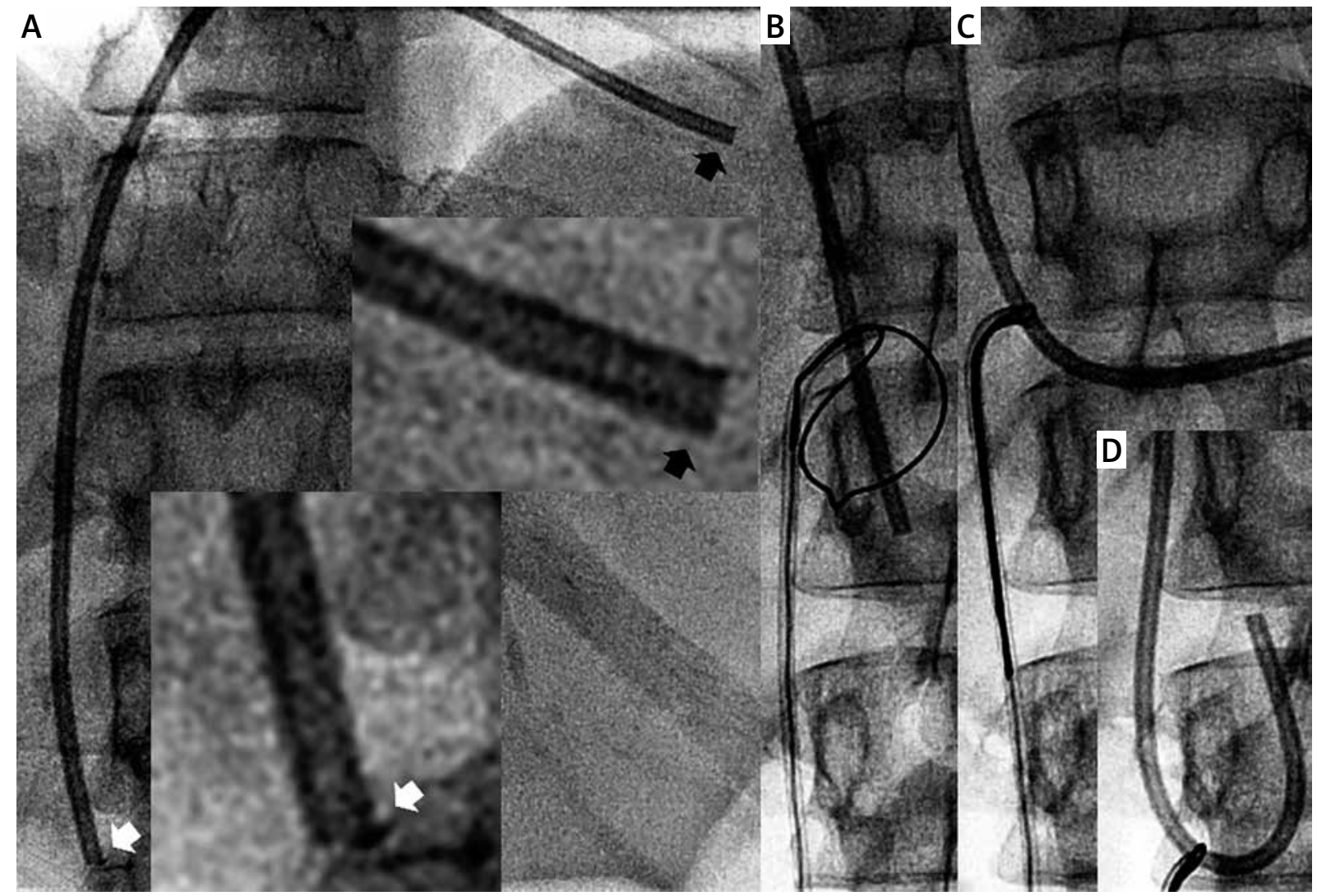

Figure 4. Another example of Polysite Mini 3000 Series catheter disconnection from the injection port implanted medially in the left infraclavicular region. The catheter's forward edge originating in the drainage area of the VCS passed through the RA and down into the $\mathrm{VCI}(\mathbf{A}$, white arrow), whereas the trailing edge passed into the RA and lodged against the RV myocardium (A, black arrow). Note that neither edge was distorted (see the magnified pictures, correspondingly). The Amplatz Nitinol goose-neck snare (B) was advanced from the right femoral vein and grasped the catheter at its distal free end (C), which was then pulled down (D)

insertion and thus allied POS (Cath-Lock mechanism) [2, 3]. It is also proposed to tie a suture around the proximal part of the catheter at its entrance, to fix it securely. The real incidence of CVC catheter fragment embolization is claimed to be higher than reported, and it occurs often as a complication of catheter extraction, due to its breakage or separation from the hub, or due to severing while cutting the fixation suture $[5,6]$. It could also result from the POS, even if the CVC is inserted into the internal jugular vein [16-18]. Currently documented anatomic sites of the foreign body lodgment are similar to those reported previously, and depend on the catheter's length, weight, and the material stiffness, with the unusual location, e.g. in the left atrium via the atrial septal defect $[3,19]$. In 1970 Bernhardt et al., for the first time, reviewed American literature and identified several case reports describing the clinical after-effects of intracardiac catheter embolization. Remarkably, out of a total of 62 subjects studied, there were 28 in whom removal was not done, and 17 of those (61\%) died of related sepsis, perforation, thrombosis, arrhythmias or myocardial necrosis, whereas there were no deaths among the other 34 subjects in whom removal was performed, either surgically (in the majority) or using a non-surgical technique [20]. In another large scale review of 220 documented cases of catheter embolism, the associated morbidity was $71 \%$ and the mortality $38 \%$, when a catheter fragment was not removed [21]. Interestingly, it was shown that the catheter piece could bounce to and fro on the tricuspid valve, with its ends making whipping motions, which potentially could cause mechanical damage to the valve and initiate endocarditis even in the absence of preexisting valvular disease [22]. Whereas the literature clearly advocates early extraction after an acute occurrence of catheter embolization, there are reports of an incidental finding of catheter fragments after an uneventful and an asymptomatic period of 9 or 14 years since embolization $[23,24]$. At the same time, late percutaneous retrievals performed in a chronic setting years after embolization in asymptomatic patients were proved to be feasible [25, 26]. Patients with embolized foreign bodies are often in a poor general condition and are particularly vulnerable 


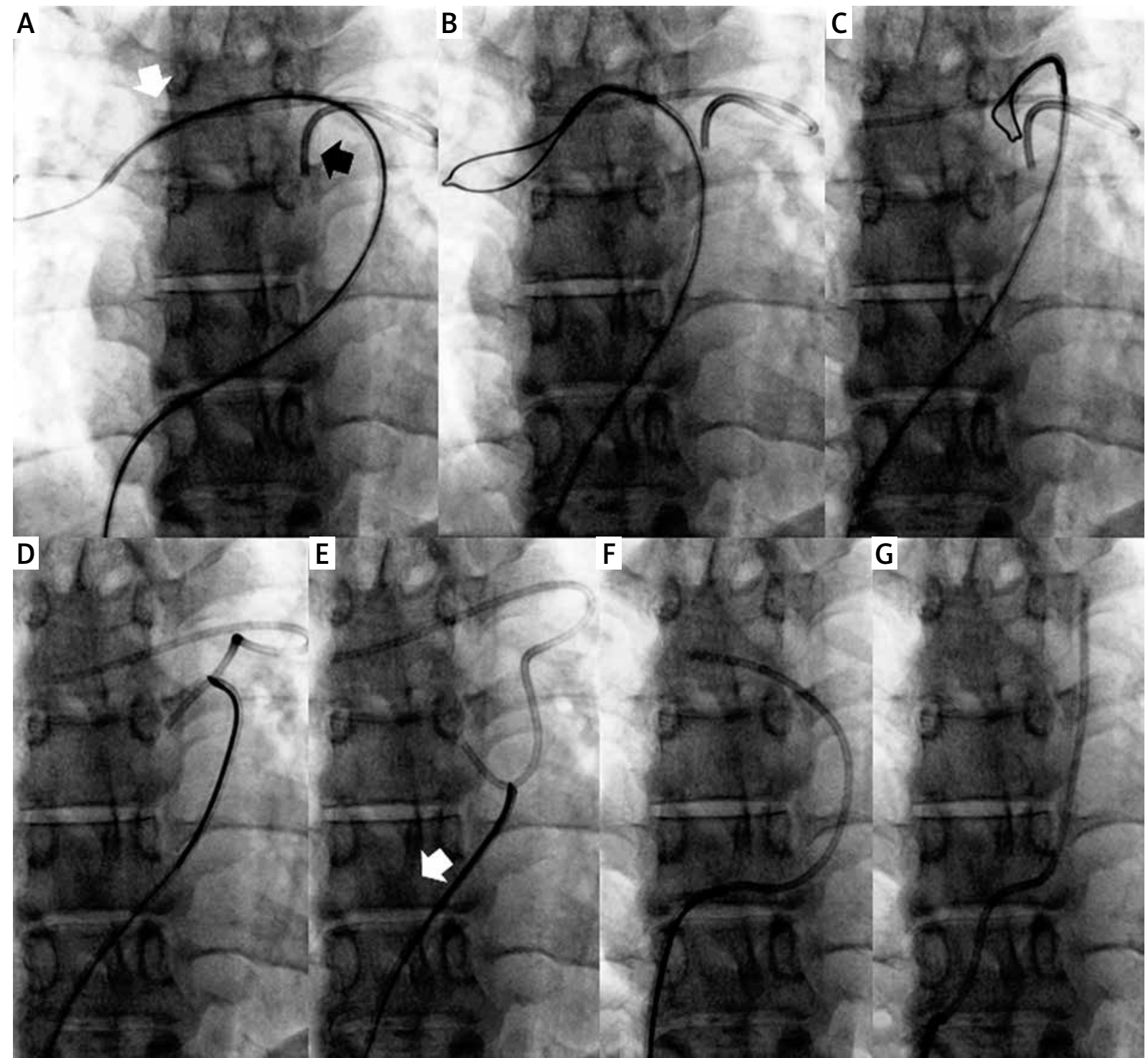

Figure 5. The $17.0 \mathrm{~cm}$ long Polysite Mini 3000 Series catheter fragment became disconnected and lodged entirely in the PA, with its forward edge located in the right main pulmonary branch (white arrow, A) and the trailing edge hanging downward in the pulmonary trunk, just above the pulmonary valve leaflets (black arrow, A). The forward edge was free floating and of favorable position, therefore allowing the Amplatz Nitinol gooseneck snare to properly expand and successfully ensnare the catheter fragment (B and $\mathbf{C})$. Then the grasped catheter was pulled down across RV outflow tract (D and $\mathbf{E}$ ), then the tricuspid valve (F) and finally the $\mathrm{VCI}(\mathbf{G})$

to sepsis or thrombosis. Thoracotomy and open heart major surgery for cardiac foreign body removal are by definition more invasive, time consuming, and expensive than percutaneous retrieval and were reported to carry a mortality rate of $10 \%$. However, no deaths or major complications have been reported as a result of percutaneous removal of an embolized catheter, with the success rate ranging from $87 \%$ to $98 \%[5,15,27,28]$. A large variety of techniques and devices for intravascular non-surgical foreign bodies retrieval are available, including wire snares, Dormia baskets, hook-shaped catheters and grasping forceps. Today wire snares are the most commonly used and successful if there is a free-floating catheter edge and enough space to expand the loop (Figures $2-5,10,12$ ). If the ends of the embolized fragment are firmly wedged in the myocardial wall, preliminary maneuvers are performed to orient the object in a manner suitable for snaring, usually with a pigtail catheter. Of course, the possibility that the object may migrate from a safer site to a more tortuous and complicated course exists (Figures $6,9,11,13,14$ ) [29]. In the case of absence of dedicated wire snares, physicians familiar with catheterization procedures might use a double-over GW (0.014 inch) and place it inside the regular angiographic catheter through its proximal lumen (even diagnostic catheters can be used providing they have a 0.035 inch 


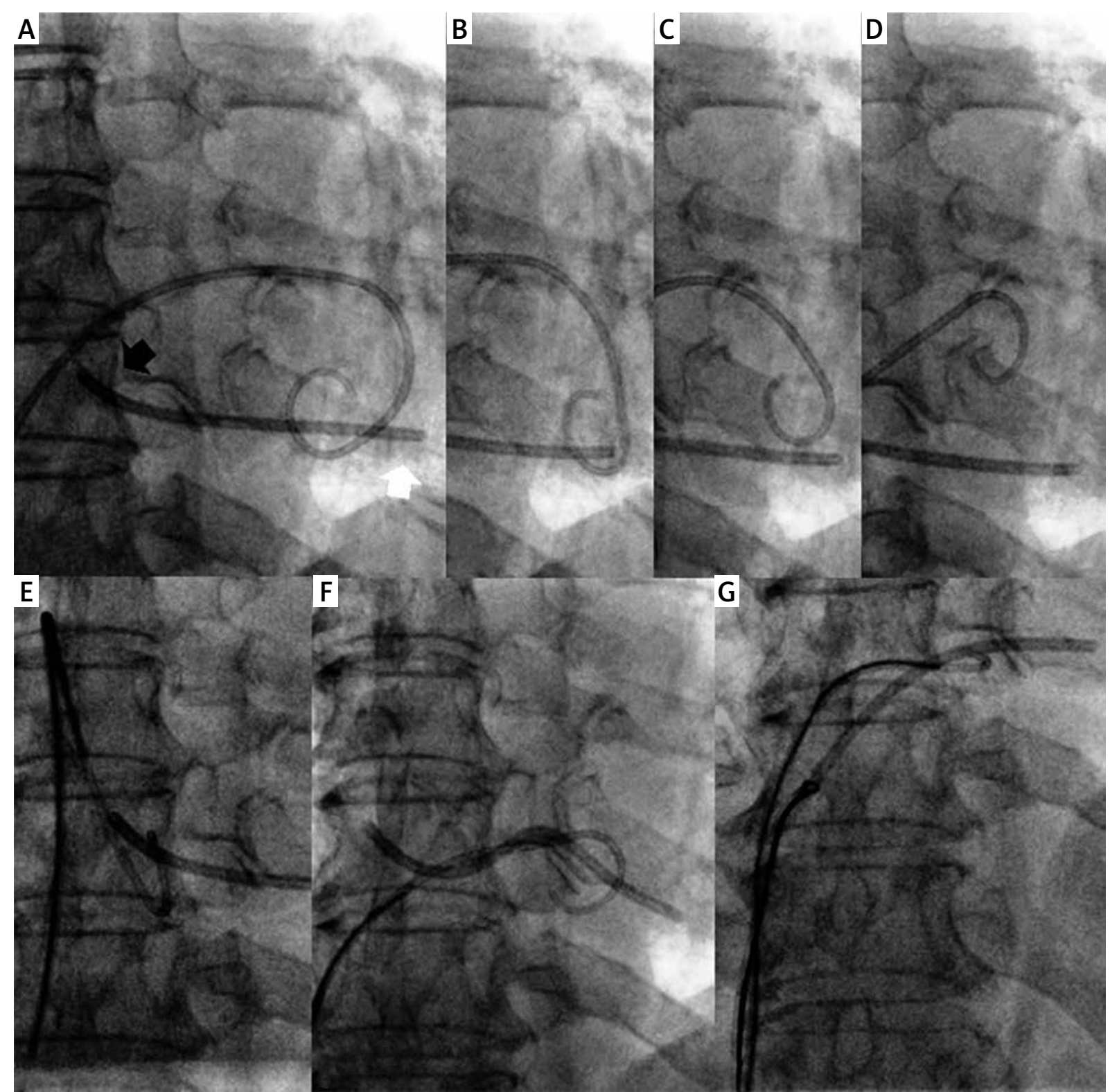

Figure 6. An embolized 83-mm long catheter fragment of Celsite Access Port, with both its lodging sites found in the right heart. Both edges were inaccessible for the snare, with the forward edge firmly impinged in the RV apex (white arrow) and the trailing edge lying anteriorly in the low RA (black arrow). Therefore, a 6 Fr pigtail catheter was used to reposition the forward fragment's edge; however, this encirclement turned out to fail (A-D). Then, it was successfully maneuvered to wind around the opposite, trailing catheter's edge, liberating it into the $\mathrm{VCl}$, where it was easily snared $(\mathrm{E}-\mathrm{G})$

internal diameter), then selectively retract one end of the GW protruding from the proximal lumen of the introducing catheter, sliding a movable metal wire in and out of the distal end of the catheter [30]. Interestingly, in order to guarantee a wide circular sweep, and to avoid a tight angle formation of a diamond shape of a midsection of a guide-wire folded in half, it should be inserted into the guiding catheter the other way; both ends of the GW should be introduced into the distal end of the catheter and advanced until the circular loop of the GW band projects from the patient end of the catheter [31]. The disad- vantage of the above homemade snares is that they frequently open parallel and adjacent to the foreign body, requiring multiple attempts to deflect the snare around the catheter fragment. Catheters with a curved tip might help to overcome this disadvantage [32]. In 1971 Dotter et al. removed the errant $8 \mathrm{~cm}$ long polyethylene CVC catheter from an RA using a homemade snare. Due to failed attempts to ensnare the catheter fragment with a single loop, the authors advanced several inches of thin tubing into the RA and thus created a complex, compound and convoluted loop, which facilitated catching 

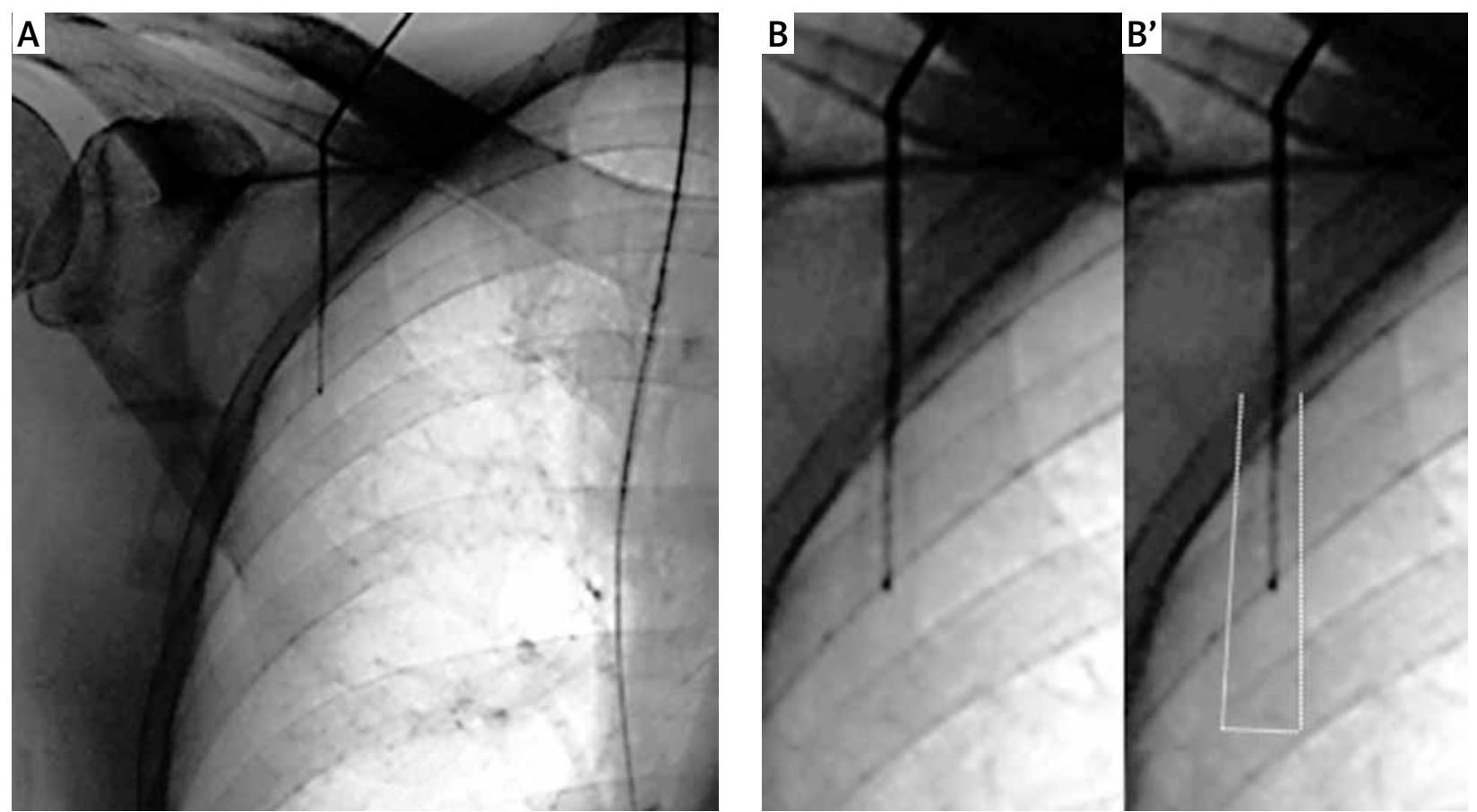

Figure 7. An incident of inadvertent GW loss during Hydrocath Assure CVC percutaneous insertion into the right internal jugular vein using the Seldinger technique. The patient was immediately referred for percutaneous extraction of the GW, but CXR (A) revealed that the proximal GW tip was located only a few millimeters inside the connector entrance (magnified B and B'). Therefore the CVC hub was cut flush and the guide-wire was grasped with forceps and easily retrieved

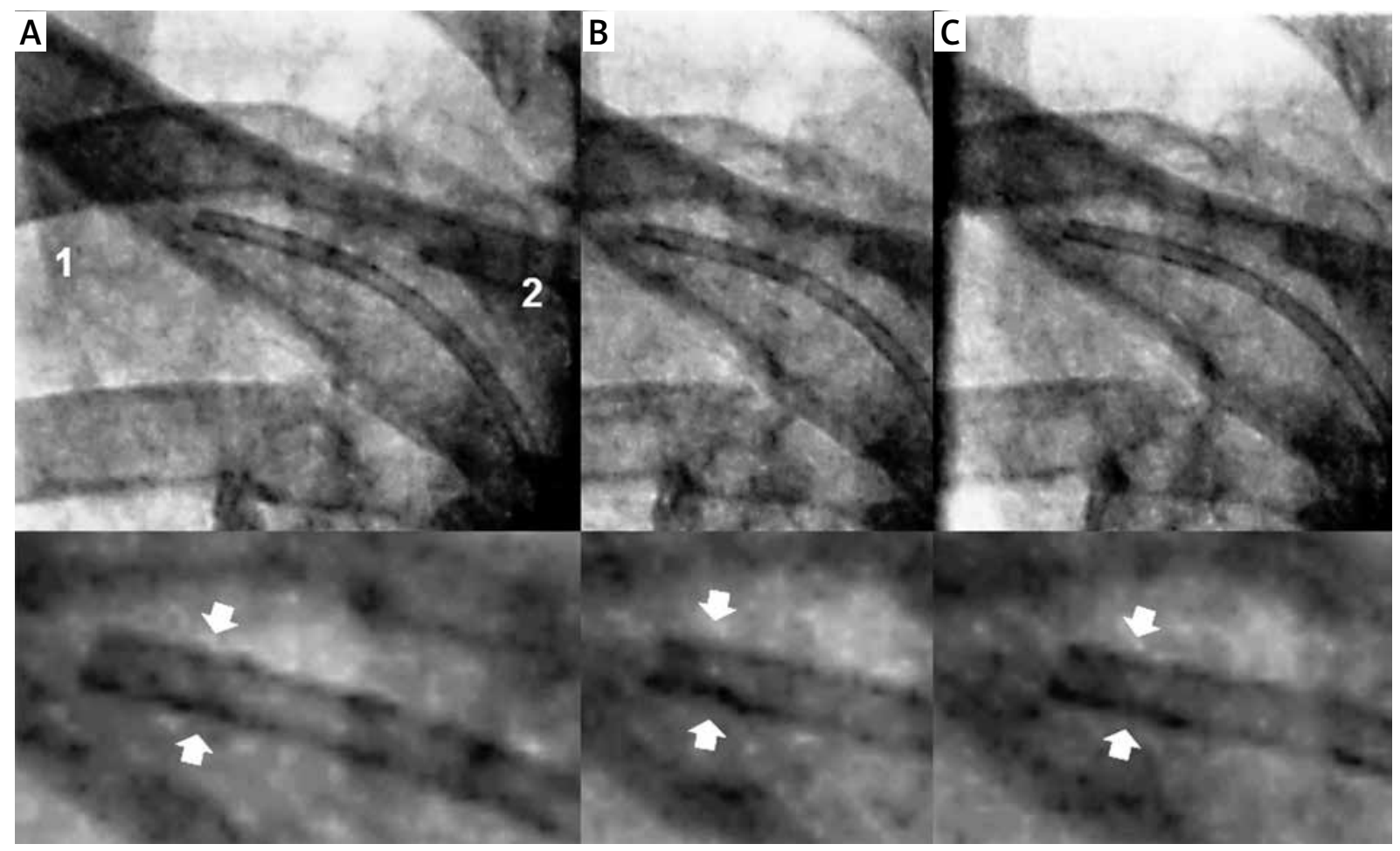

Figure 8. Radiologic sign of the POS syndrome sequel. Note the Broviac Expert CVC's catheter transection located exactly in the area of the $1^{\text {st }}$ right rib (1) and near the right clavicle (2), signifying the occurrence of catheter compression leading to its distortion (white arrows) and subsequent rupture. In the upper row are presented the 3 consecutive angiographies $(A-C)$ taken during the catheter percutaneous removal. Correspondingly in the lower row are presented the same but magnified pictures of the catheter's edge, which is indented $(2.5 \times z 00 \mathrm{~m})$ 

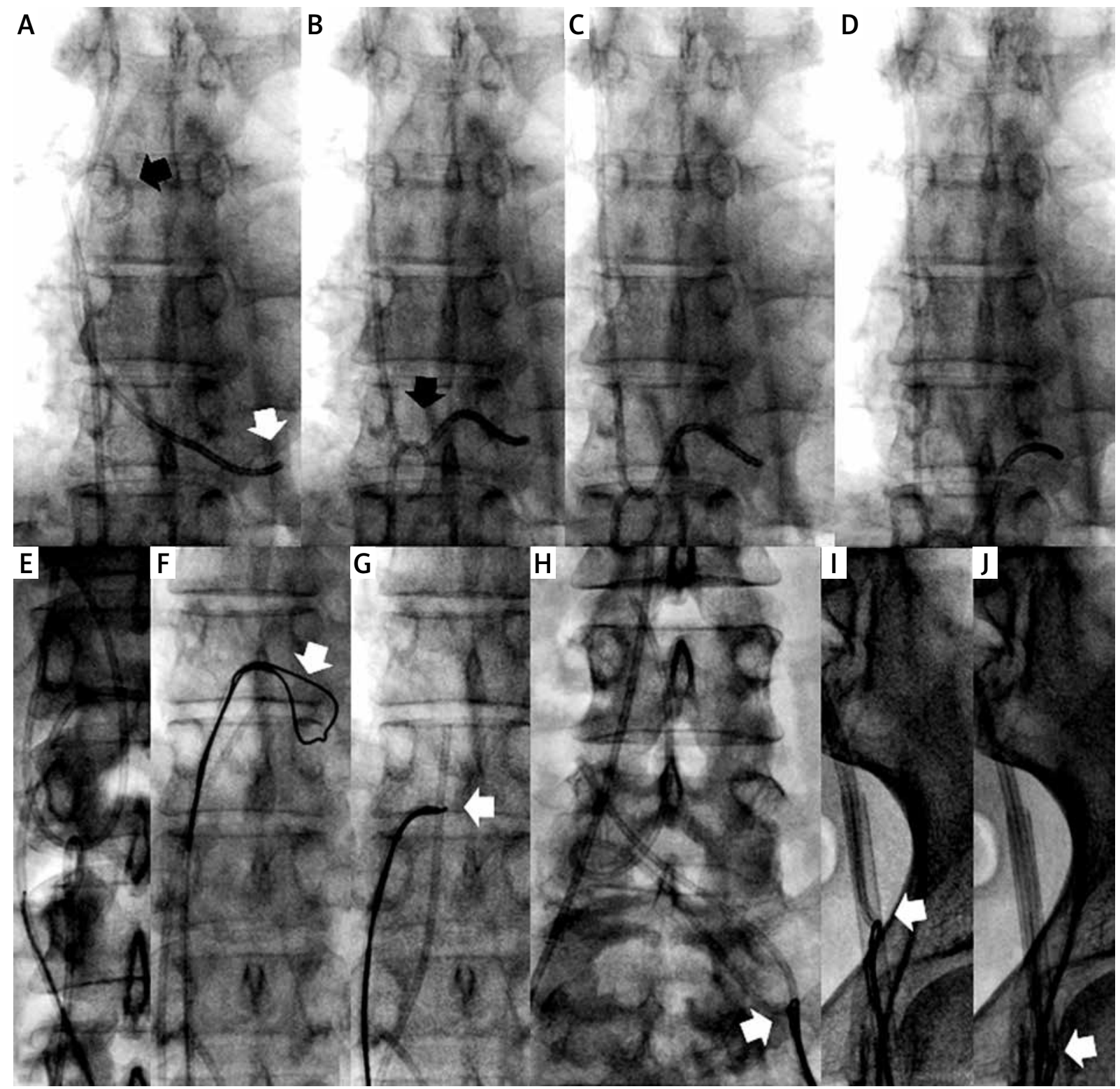

Figure 9. Broviac CVC catheter fragment of $19 \mathrm{~cm}$ length, with its intracardiac lodging site in the inferior wall of the RV myocardium (forward edge, white arrow) and its trailing edge found in the right subclavian vein (Figure 3). A $5 \mathrm{Fr}$ pigtail catheter was introduced from the right femoral vein into the VCS (black arrow) and hooked the catheter fragment (black arrow), which was pulled down, liberating the catheter's forward edge (B-E). Then, the Amplatz Nitinol goose-neck snare, which is of a right angle design ( $F$, white arrow), was inserted transfemorally but contralateral and easily encircled the catheter's free-end, which was pulled down inside the $14 \mathrm{Fr}$ vascular sheath (F-J)

the foreign body. The authors postulated that the design and manner of homemade assemblies are tailored to the specific problem and could be constructed on an ad hoc basis, using materials ordinarily at hand [12]. At the same time, the novel, custom-made Multi-Snare (pfm medical ag, Köln, Germany), due to its unique but simple design, offers the additional perpendicular plane of sweep. A major advantage of forceps (endomyocardial bioptome) with their grasping capability over snares or baskets is the ability to seize a foreign body at its middle portion, which eliminates the need for repositioning [19]. Discordant with our experience, a few authors postulate that it is important to avoid the snared foreign body being exposed 'bare' to the RV cavity with its tortuous outflow tract. In their opinion, the foreign body could otherwise lodge in the intraventricular structures [33]. This could be prevented by using long trans-septal sheaths of a sufficient gauge (8 or $11 \mathrm{Fr}$ ), positioned as close as possible to the embolized device, which is finally drawn completely into the sheath [34]. Also, this could help to negotiate the 

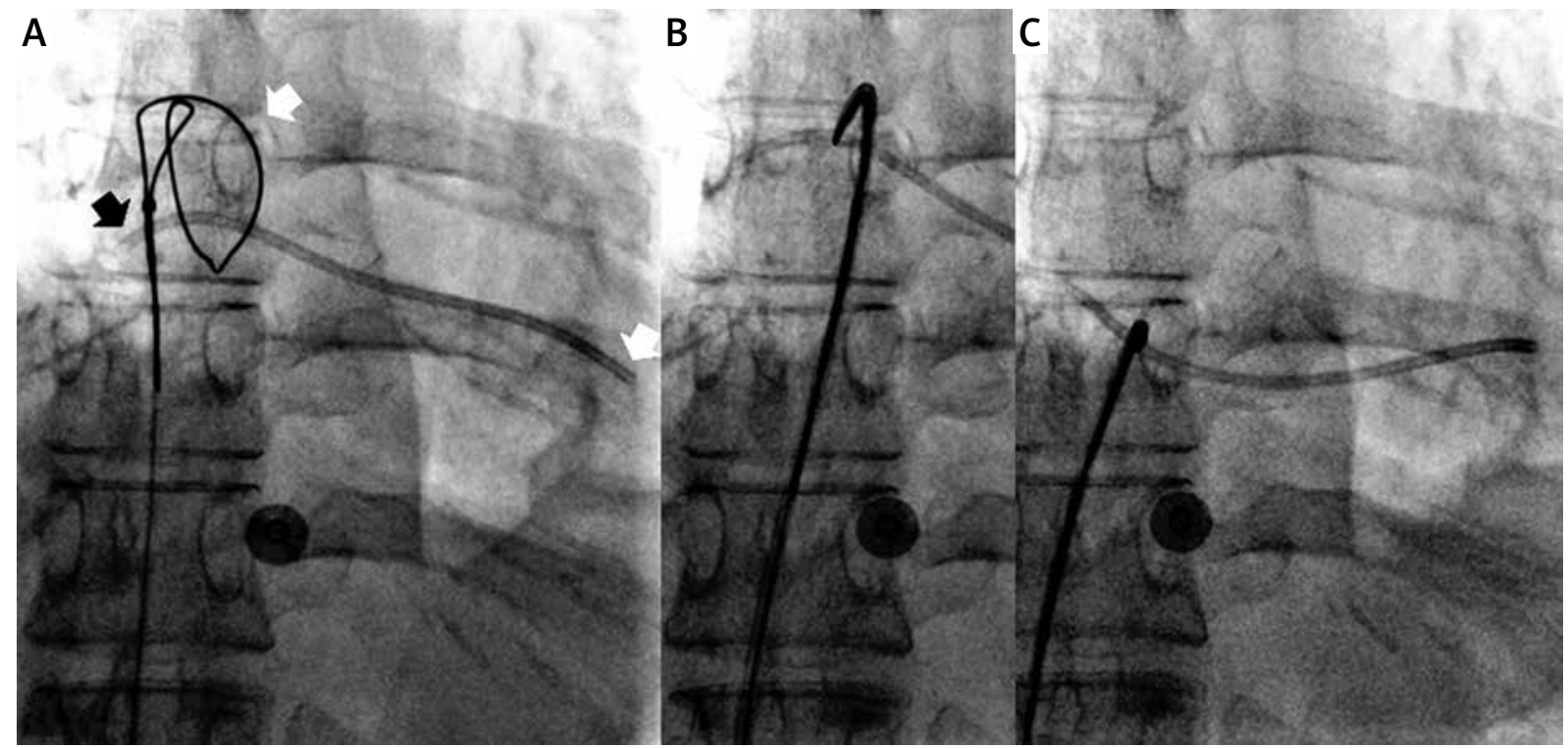

Figure 10. Angiographic appearance of the intracardiac lodging site and 85-mm length of the migrated Ambix Intraport catheter fragment are both similar to the respective features presented in Figure 6 . But, contrary to the one presented earlier, the loop retriever alone with its operational right angle design (white arrow) successfully snared the catheter $(B-C)$
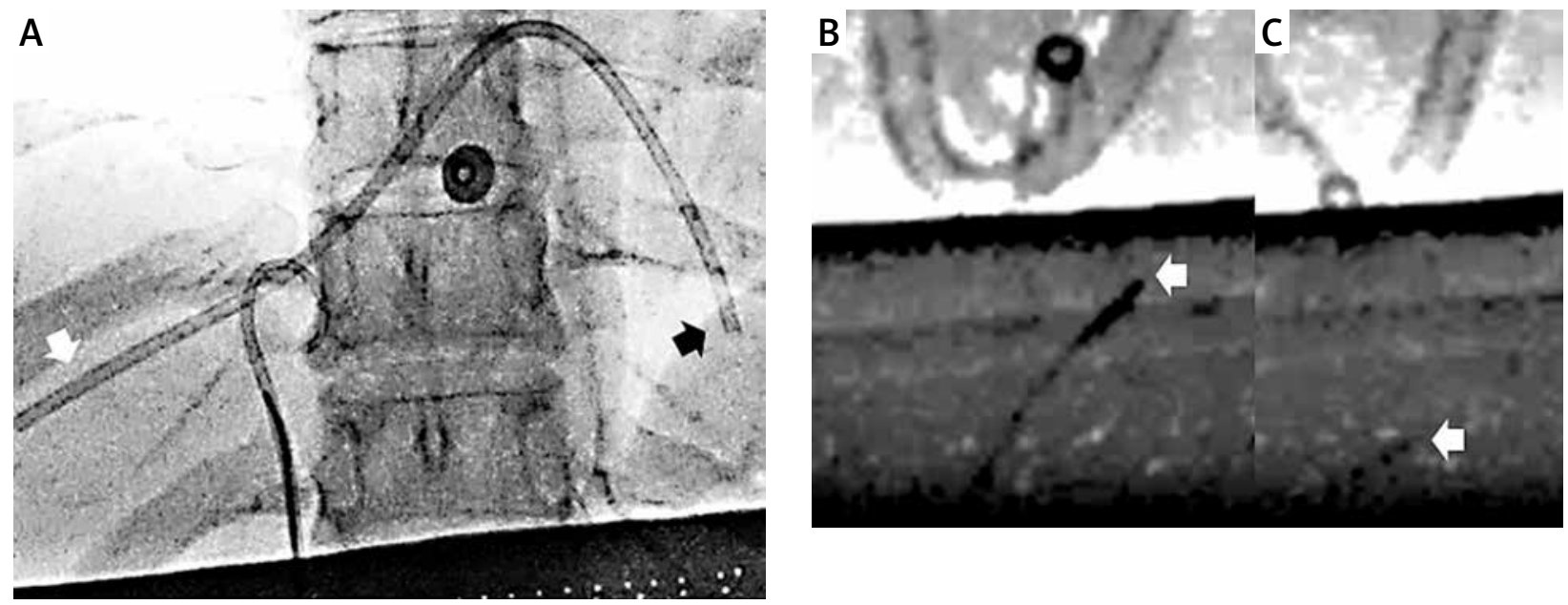

Figure 11. The 156-mm long PowerPort BARD catheter fragment lodged with its forward edge in the RV (white arrow) and its trailing edge migrated in the hepatic vein (black arrow). Both edges were inaccessible for the snare. A 6 Fr pigtail catheter hooked the embolized catheter fragment at its mid portion and both edges were still firmly impinged at their prime localizations. Therefore, the loop retriever $(20-\mathrm{mm} \emptyset)$ was used to snare the tip of the pigtail catheter. The foreign body was pulled tightly against the sheath, and thereafter the whole assembly was pulled down and withdrawn as a unit (A-C)

tortuous course of the RV cavity and its outflow tract when using rigid baskets or forceps. Finally, "the double wire technique" during the retrieval procedures is sometimes recommended. In this method, after a vascular sheath is placed, a second GW is advanced through it into the vessel. Then, the sheath is withdrawn and reinserted over only one of the two wires. The sheath thus contains one wire and sits adjacent to the one wire, which acts at the same time as a "buddy wire" and "safety wire". This arrangement helps to minimize potential injury to the internal vascular wall during the removal of a whole sheath-snared-catheter complex, and also allows immediate reentry of the vessel over the second wire (Figure 3) [35]. We agree that continuous fluoroscopic guidance is necessary during all retrieval procedures, especially during the moment when the snared catheter fragment is pulled down and withdrawn, to avoid its unexpected kinking. The mean length of the embolized catheter fragments reported in the literature $(14.3 \pm 2.6 \mathrm{~cm})$ is similar to our results, along with concor- 
A

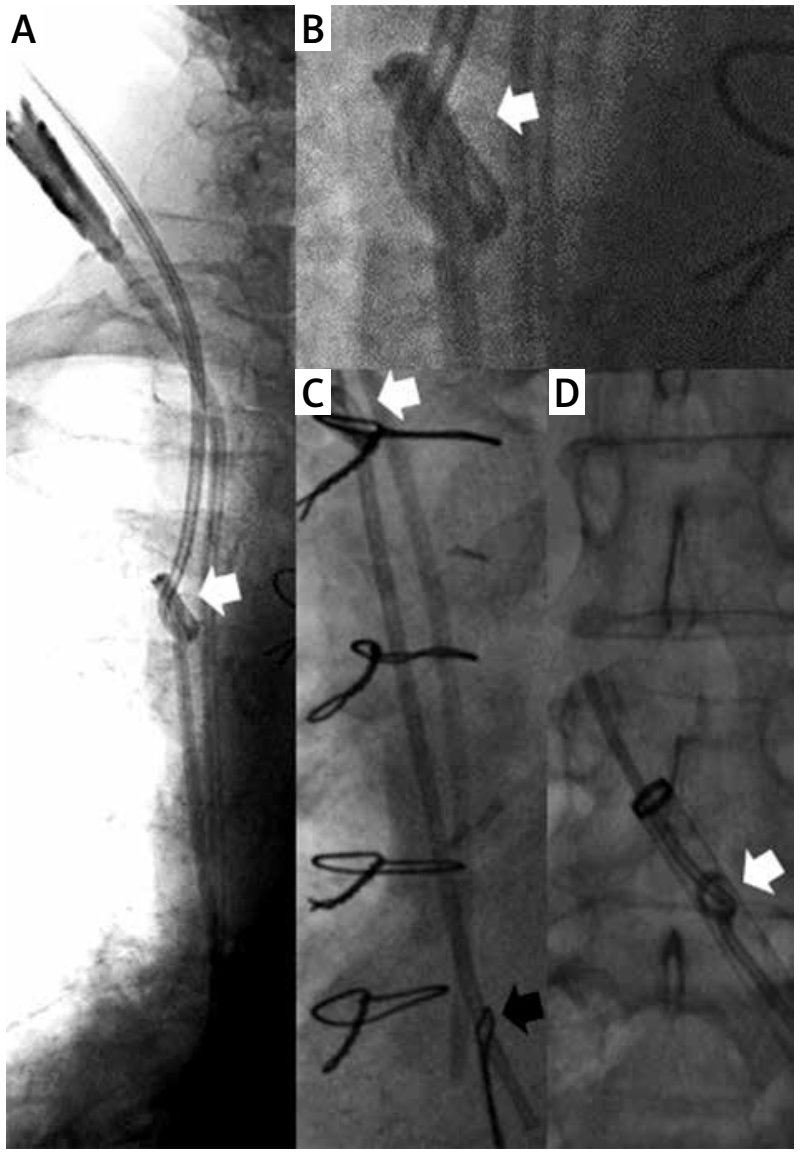

Figure 12. An example of a knotted SG catheter placed in the right internal jugular vein (inverse mode of digital CXR, A). A tight knot is located in the VCS. The snare which was introduced transfemorally easily encircled the SG's proximal edge. Then, the catheter was cut at its entrance and subsequently pulled down as a whole with the snare inside the $14 \mathrm{Fr}$ vascular sheath. Finally, it was withdrawn outside through the sheath's hub (B-D) dant mean procedure and fluoroscopy times, as well as DAP values [3, 13, 36].

A limitation of our study is that neither the actual time of the insertion and thus duration of the interval between the dislodgment and percutaneous retrieval of the foreign body, nor the cause of catheter embolization in each studied subject, could be determined. In conclusion, by using the pigtail catheter and the loop snare it is feasible to retrieve centrally embolized fragments or knotted central venous access devices.

\section{Acknowledgments}

We thank Ms. Katarzyna Szcześniak for her invaluable assistance with English proofreading and editing services.

\section{Conflict of interest}

The authors declare no conflict of interest.

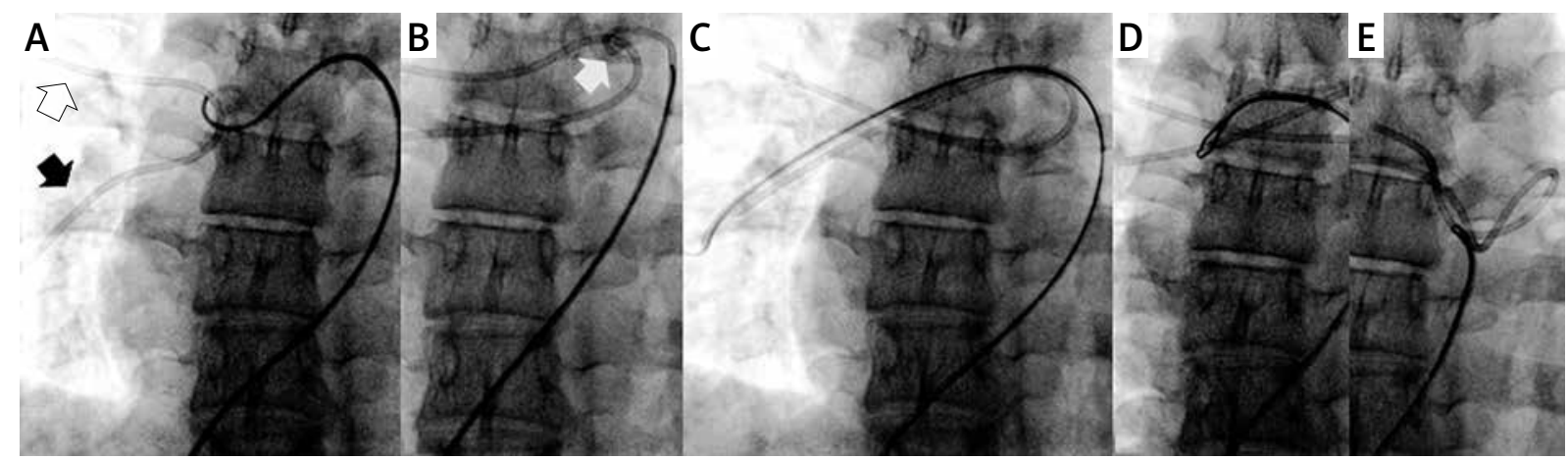

Figure 13. The 184-mm long fragment of PowerPort BARD catheter embolized distally into the right PA, with one of its edges firmly lodged in the ascending branch of the PA (white arrow) and the other lodged in its descending branch (black arrow). Therefore, a $6 \mathrm{Fr}$ pigtail catheter elegantly hooked the mid segment of the migrated fragment (grey arrow) and was pulled toward the left side, liberating both edges (B). Then the pigtail catheter was substituted with a JR4 guiding catheter, which was advanced into the descending branch of the PA, allowing the loop to be extended and to encircle the free end of the catheter fragment, with its final successful retrieval (D and $\mathbf{E})$ 

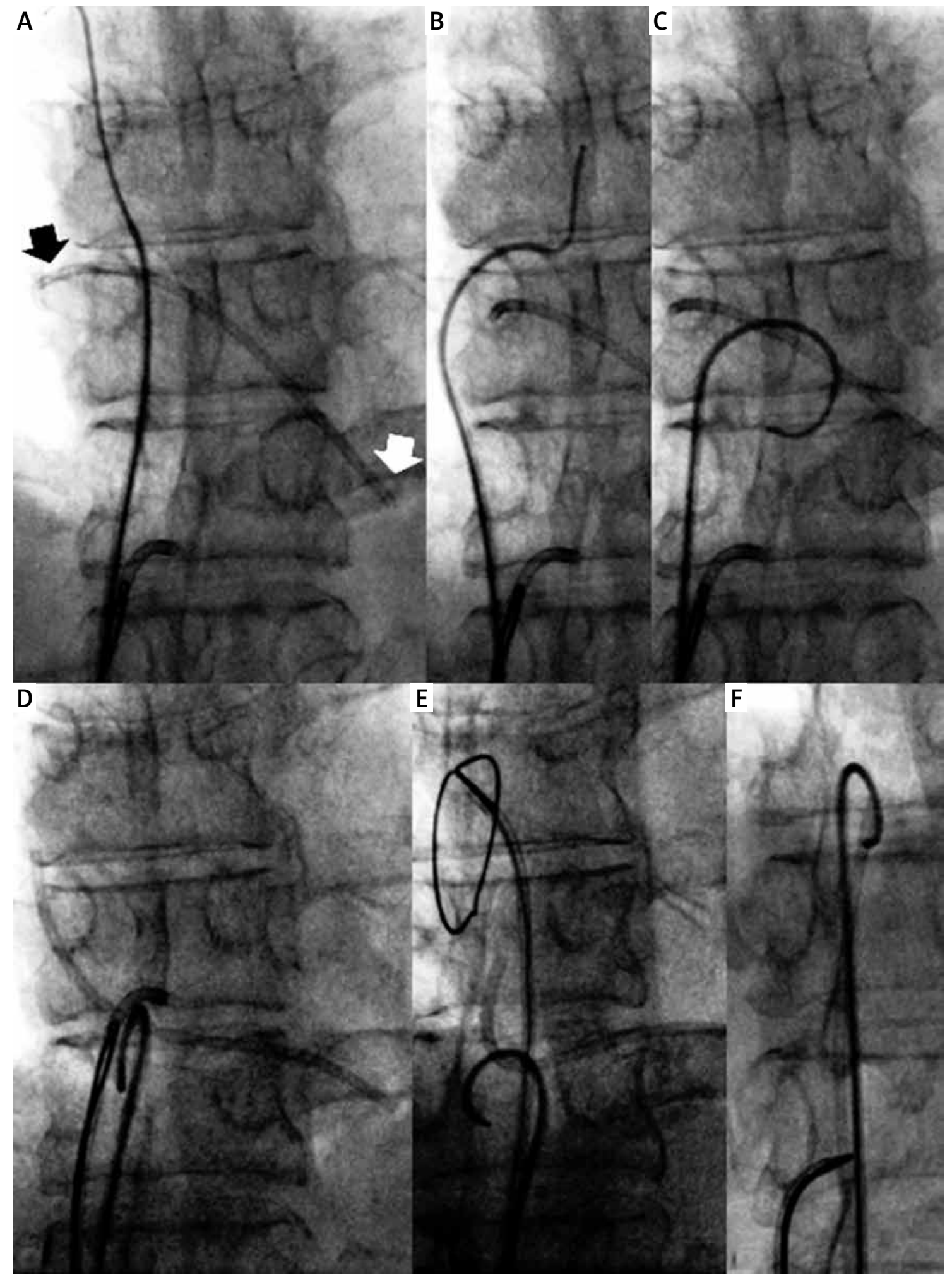

Figure 14. One of the shortest presented, a 69-mm long Ambix Intraport catheter fragment, was entirely wedged in the RA, with its forward edge lodged in the tricuspid annulus (white arrow) and its trailing edge lying anteriorly in the mid RA (black arrow). A 6 Fr pigtail catheter was passed above the migrated catheter's fragment, along the $0.889-\mathrm{cm}$ (0.035 inch) GW, which was advanced into the VCS. Then, the GW was withdrawn so as to bend the pigtail curve, which firmly hooked the catheter's mid-shaft. Then the pigtail catheter was pulled down, releasing the trailing edge of the migrated catheter, allowing its successful ensnarement and subsequent entire catheter retrieval 


\section{References}

1. Kock HJ, Pietsch M, Krause U, et al. Implantable vascular access systems: experience in 1500 patients with totally implanted central venous port systems. World J Surg 1998; 22: 12-6.

2. Lin $\mathrm{CH}, \mathrm{Wu} \mathrm{HS}$, Chan DC, et al. The mechanisms of failure of totally implantable central venous access system: analysis of 73 cases with fracture of catheter. Eur I Surg Oncol 2010; 36: 100-3.

3. Liu JC, Tseng HS, Chen CY, et al. Percutaneous retrieval of 20 centrally dislodged Port-A catheter fragments. Clin Imaging 2004; 28: 223-9.

4. Kusminsky RE. Complications of central venous catheterization. J Am Coll Surg 2007; 204: 681-96.

5. Surov A, Wienke A, Carter JM, et al. Intravascular embolization of venous catheter--causes, clinical signs, and management: a systematic review. JPEN J Parenter Enteral Nutr 2009; 33: 677-85.

6. Gabelmann A, Kramer S, Gorich J. Percutaneous retrieval of lost or misplaced intravascular objects. AJR Am J Roentgenol 2001; 176: 1509-13.

7. Kuter DJ. Thrombotic complications of central venous catheters in cancer patients. Oncologist 2004; 9: 207-16.

8. Chuang MT, Wu DK, Chang CA, et al. Concurrent use of pigtail and loop snare catheters for percutaneous retrieval of dislodged central venous port catheter. Kaohsiung J Med Sci 2011; 27: 514-9.

9. Karanikas ID, Polychronidis A, Vrachatis A, et al. Removal of knotted intravascular devices. Case report and review of the literature. Eur J Vasc Endovasc Surg 2002; 23: 189-94.

10. Druskin MS, Siegel PD. Bacterial contamination of indwelling intravenous polyethylene catheters. JAMA 1963; 185: 966-8.

11. Savage C, Ozkan OS, Walser EM, et al. Percutaneous retrieval of chronic intravascular foreign bodies. Cardiovasc Intervent Radiol 2003; 26: 440-2.

12. Dotter CT, Rösch J, Bilbao MK. Transluminal extraction of catheter and guide fragments from the heart and great vessels: 29 collected cases. Am J Roentgenol Radium Ther Nucl Med 1971; 111: 467-72.

13. Aitken DR, Minton JP. The "pinch-off sign": a warning of impending problems with permanent subclavian catheters. Am J Surg 1984; 148: 633-6.

14. Hinke DH, Zandt-Stastny DA, Goodman LR, et al. Pinch-off syndrome: a complication of implantable subclavian venous access devices. Radiology 1990; 177: 353-6.

15. Cheng CC, Tsai TN, Yang CC, et al. Percutaneous retrieval of dislodged totally implantable central venous access system in 92 cases: experience in a single hospital. Eur J Radiol 2009; 69: 346-50.

16. Aroesty JM, Cohen SI. Traction-induced fracture of a central venous pressure catheter. Chest 1971; 60: 515-6.

17. Scott WL. Complications associated with central venous catheters. A survey. Chest 1988; 94: 1221-4.

18. Shimizu A, Lefor A, Nakata M, et al. Embolization of a fractured central venous catheter placed using the internal jugular approach. Int J Surg Case Rep 2014; 5: 219-21.

19. Bloomfield DA. Techniques of nonsurgical retrieval of iatrogenic foreign bodies from the heart. Am J Cardiol 1971; 27: 538-45.

20. Bernhardt LC, Wegner GP, Mendenhall JT. Intravenous catheter embolization to the pulmonary artery. Chest 1970; 57: 329-32.

21. Fisher RG, Ferreyro R. Evaluation of current techniques for nonsurgical removal of intravascular iatrogenic foreign bodies. AJR Am J Roentgenol 1978; 130: 541-8.
22. Mariano BP Jr, Roper CL, Staple TW. Accidental migration of an intravenous infusion catheter from the arm to the lung. Radiology 1966 ; 86 : 736-8.

23. Reynen K. 14-year follow-up of central embolization by a guidewire. N Engl J Med 1993; 329: 970-1.

24. Andrews RE, Tulloh RM, Rigby ML. Percutaneous retrieval of central venous catheter fragments. Arch Dis Child 2002; 87: 149-50.

25. Oto A, Tokgozoglu SL, Oram A, et al. Late percutaneous extraction of an intracardiac catheter fragment. Jpn Heart J 1993; 34: 117-9.

26. Thanigaraj S, Panneerselvam A, Yanos J. Retrieval of an IV catheter fragment from the pulmonary artery 11 years after embolization. Chest 2000; 117: 1209-11.

27. Schüler S, Hetzer R, Stegmann T, et al. Surgical therapy of intracardiac infected pacemaker electrodes and catheter remnants. Z Kardiol 1986; 75: 151-5.

28. Richardson JD, Grover FL, Trinkle JK. Intravenous catheter emboli. Experience with twenty cases and collective review. Am J Surg 1974; 128: 722-7.

29. Huggon IC, Qureshi SA, Reidy J, et al. Percutaneous transcatheter retrieval of misplaced therapeutic embolisation devices. $\mathrm{Br}$ Heart J 1994; 72: 470-5.

30. Massumi RA, Ross AM. Atraumatic, nonsurgical technic for removal of broken catheters from cardiac cavities. N Engl J Med 1967; 277: 195-6.

31. Hartnell GG. Homemade snare for removal of foreign bodies. Radiology 1991; 181: 903-4.

32. Curry JL. Recovery of detached intravascular catheter or guide wire fragments. A proposed method. Am J Roentgenol Radium Ther Nucl Med 1969; 105: 894-6.

33. Richardson JD, Grover FL, Trinkle JK. Intravenous catheter emboli. Experience with twenty cases and collective review. Am J Surg 1974; 128: 722-7.

34. Kohli V, Joshi R. Central line retrieval in a neonate. Indian J Pediatr 2007; 74: 692-3.

35. Dawson S, Papanicolaou N, Mueller PR, et al. Preserving access during percutaneous catheterization using a double-guide-wire technique. AJR Am J Roentgenol 1983; 141: 407.

36. Strohmer B, Altenberger J, Pichler M. A new approach of extracting embolized venous catheters using a large-diameter steerable sheath under biplane fluoroscopy. Clin Imaging 2012; 36: 502-8. 